\title{
Evaluation of Delcath Systems' Generation 2 (GEN 2) Melphalan Hemofiltration System in a Porcine Model of Percutaneous Hepatic Perfusion
}

\author{
Fred M. Moeslein • Elizabeth G. McAndrew • William M. Appling • \\ Nicole E. Hryniewich · Kevin D. Jarvis · Steven M. Markos · Timothy P. Sheets • \\ Rajneesh P. Uzgare $\cdot$ Daniel S. Johnston
}

Received: 18 July 2013 / Accepted: 7 December 2013/Published online: 9 January 2014

(c) The Author(s) 2014. This article is published with open access at Springerlink.com

\begin{abstract}
Purpose A new melphalan hemoperfusion filter (GEN 2) was evaluated in a simulated-use porcine model of percutaneous hepatic perfusion (PHP). The current study evaluated melphalan filtration efficiency, the transfilter pressure gradient, and the removal of specific blood products.

Materials and Methods A porcine PHP procedure using the GEN 2 filter was performed under Good Laboratory Practice conditions to model the 60-min clinical PHP procedure.

Results The mean filter efficiency for removing melphalan in six filters was $99.0 \pm 0.4 \%$. The transfilter pressure gradient across the filter averaged $20.9 \mathrm{mmHg}$ for the 60-min procedure. Many blood components, including albumin and platelets, decreased on average from 3.55 to $2.02 \mathrm{~g} / \mathrm{dL}$ and from 342 to $177 \times 10 . \mathrm{e} 3 / \mu \mathrm{L}$, respectively, during the procedure.

Conclusion The increased melphalan extraction efficiency of the new filter is expected to decrease systemic
\end{abstract}

Electronic supplementary material The online version of this article (doi:10.1007/s00270-013-0826-5) contains supplementary material, which is available to authorized users.

\section{F. M. Moeslein}

Department of Diagnostic Radiology and Nuclear Medicine, University of Maryland School of Medicine, 22 South Greene St, Baltimore, MD 21201, USA

e-mail: fmoeslein@umm.edu

E. G. McAndrew $(\bowtie)$. W. M. Appling ·

N. E. Hryniewich · K. D. Jarvis · S. M. Markos ·

T. P. Sheets · R. P. Uzgare · D. S. Johnston

Delcath Systems, Inc., 566 Queensbury Ave., Queensbury

NY 12804, USA

e-mail: emcandrew@delcath.com

W. M. Appling

e-mail: billappling@yahoo.com melphalan exposure. In addition, the low transfilter pressure gradient resulted in low resistance to blood flow in the GEN 2 filter, and the changes to blood components are expected to be clinically manageable.

Keywords Percutaneous hepatic perfusion - Cancer . Melphalan $\cdot$ Liver $\cdot$ Chemofiltration $\cdot$ Porcine

\section{Introduction}

Regionalized and organ-specific methods for chemotherapeutic treatment limit the toxicity of systemic chemotherapy by facilitating high local drug concentrations at the site of the malignancy compared with systemic administration [1]. The liver has been a primary target for these methods for $>50$ years based on the high frequency of primary and metastatic liver cancer, the ability to clinically isolate the hepatic vasculature, and the capacity of the liver to withstand toxic insult [2]. Because liver metastases receive nutrients primarily by the hepatic arterial supply as opposed to the portal vein

\footnotetext{
N. E. Hryniewich

e-mail: nhryniewich@gmail.com

K. D. Jarvis

e-mail: kevinjarvis89@gmail.com

S. M. Markos

e-mail: stevenmmarkos@gmail.com

T. P. Sheets

e-mail: researchsci22@gmail.com

R. P. Uzgare

e-mail: ruzgare@delcath.com

D. S. Johnston

e-mail: johnston.daniels@gmail.com
} 
[3], treatment by way of the arterial system has been the primary route of administration for direct high-dose chemotherapy [4, 5]. When regionally administered high-dose chemotherapy is paired with high-efficiency drug filtration of venous outflow, extraregional systemic exposure can be further minimized [4, 6]. This approach forms the basis for percutaneous hepatic perfusion (PHP) (see Fig. 1).

In PHP, filtration efficiency is one of the primary determinants of systemic exposure, and improvements in the filtration system are expected to decrease the amount of drug circulating in the body [4]. To that end, a new filter was recently developed (GEN 2 filter; Delcath Systems, New York, NY, USA), which showed improved filtration efficiency during preclinical in vitro testing. We hypothesized that during in vivo preclinical studies, this filter would have improved filtration efficiency compared with previous filtration systems used during clinical trials [4]. The new filter was evaluated in a porcine model of PHP using melphalan hydrochloride to evaluate filtration efficiency in an in vivo setting before use in the clinical setting. Melphalan filtration efficiency, systemic exposure, transfilter pressure gradient, and the effect of hemofiltration on blood parameters were evaluated.

\section{Materials and Methods}

The protocol for this study was reviewed and approved by an Institutional Animal Care and Use Committee at the testing site where the study was performed. The study was performed in September 2011. A board certified interventional radiologist and perfusionist performed all procedures with appropriately trained scientists and veterinary technicians.

Animals and preoperative care as follows: Six Yorkshire Cross pigs (5 months of age, approximately $78-91 \mathrm{~kg}$ ) were treated by way of PHP procedure with $220 \mathrm{mg}$ of melphalan hydrochloride. Animals were acclimated to the facility for a minimum of $72 \mathrm{~h}$ before the study. Food was withheld approximately $12-24 \mathrm{~h}$ before surgery. Water was withheld the morning of surgery. General anesthesia was induced, and a cuffed endotracheal tube was inserted. An intravenous catheter was placed in a vein of the right ear for fluid and heparin administration. General anesthesia was maintained with isoflurane delivered in oxygen, and a ventilator was used to assist respiration.

\section{Surgical Procedure}

Using standard cut-down techniques, an introducer sheath was placed in the femoral vein for insertion of the double-balloon

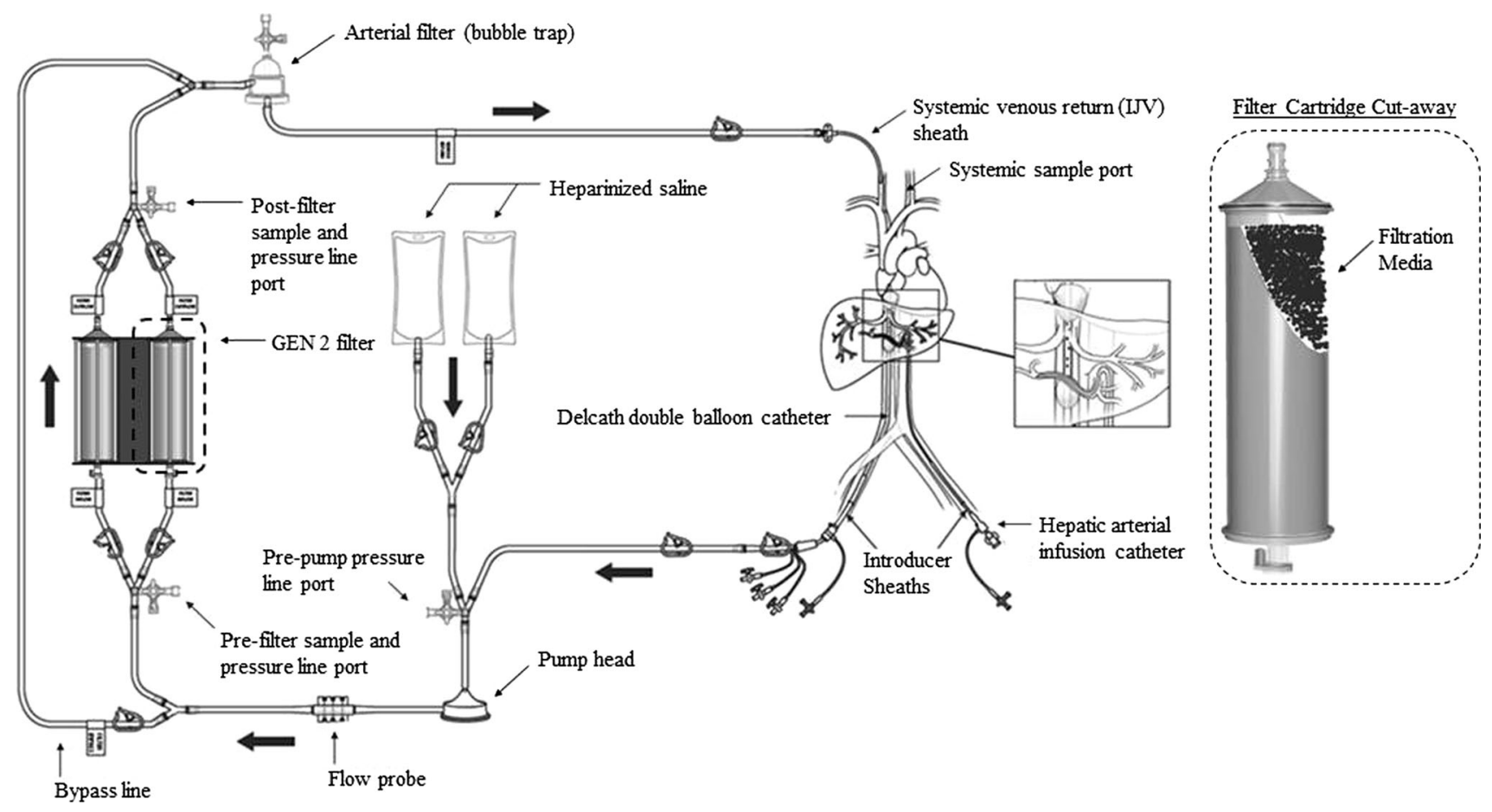

Fig. 1 Schematic of the PHP procedure. Chemotherapeutic is delivered through an infusion catheter placed in the hepatic artery. A double-balloon catheter is placed in the IVC to isolate the hepatic veins. Fenestrations between the balloons aspirate venous hepatic blood into the extracorporeal circuit (EC) through Delcath's hemofiltration cartridges. An arterial filter acts as a bubble trap to ensure that no air embolisms return with the cleaned blood into the jugular vein. A schematic of a filter cartridge is shown on the right with a cut-out to show the filtration media within 
catheter (DBC), into the femoral artery for insertion of the hepatic arterial catheter (HAC) and monitoring of intra-arterial blood pressure, into the jugular vein for blood return, and into the carotid artery for systemic arterial blood sampling. Once the sheaths were placed, $\sim 35,000 \mathrm{U}$ of heparin was administered. Coagulation was assessed by activated clotting time (ACT) approximately every $15 \mathrm{~min}$ with a target of $400 \mathrm{~s}$, and additional heparin (for a total of $35,000 \mathrm{U}$ to 75,000 U/animal) was given as needed throughout the procedure to maintain an ACT of $>400 \mathrm{~s}$.

Using fluoroscopic guidance, the HAC was placed beyond the gastroduodenal artery. The DBC was positioned in the IVC with the tip at the level of the diaphragmatic hiatus. The DBC was connected to the extracorporeal circuit (EC), which was primed with heparinized saline $(2 \mathrm{U} / \mathrm{mL})$, and the venous return sheath was connected to the perfusion adapter. The entire system was purged of air using heparinized saline.

The two balloons of the DBC were inflated with dilute contrast media so the cephalad balloon, which was inflated first, occluded the inferior vena cava (IVC) above the highest hepatic vein and the caudal balloon occluded the IVC below the lowest hepatic vein. Blood flowed through the fenestrations of the DBC into the catheter, to the EC, to the pump through the filter bypass line, and returned to the animal by way of the venous return sheath. The bypass line was then clamped, and blood was directed through the filter. The EC flow rate was adjusted to between 400 and $750 \mathrm{~mL} / \mathrm{min}$ according to the manufacturer's instructions for use.

Three minutes after the filter was introduced into the system, $220 \mathrm{mg}$ of melphalan hydrochloride in $500 \mathrm{~mL}$ of drug diluent and $0.9 \%$ saline was administered through the HAC during a 30-min "infusion period." After the infusion of drug, hemofiltration continued for a 30-min "washout period."

Blood samples were taken to measure melphalan pharmacokinetics (PK). Because the hemofiltration cartridges adsorb nonspecifically, clinical chemistry and hematology samples were taken to evaluate the impact of the procedure on blood chemistry and constituents. Baseline chemistry, coagulation, and hematology sample was obtained immediately after the systemic port was placed and at 6-min intervals throughout the procedure starting from the beginning of hemofiltration. The following blood parameters were analyzed: hematocrit, platelet count, neutrophils, albumin, and fibrinogen. A baseline systemic PK sample was taken immediately before melphalan infusion. Prefilter, postfilter, and systemic samples were taken at 3-min intervals throughout the procedure.

The animals were euthanized under general anesthesia at the end of the procedure. The primary goal of this study was to evaluate filtration efficiency; hence, tissue or organ samples were not collected for clinical pathology. The average time for each treatment was $\sim 2.5 \mathrm{~h}$.

\section{Sample Evaluation and Analysis}

Chemistry, coagulation, and hematology samples were analyzed at Physicians Reference Laboratory (Overland Park, KS, USA). Plasma melphalan concentrations were measured using liquid chromatography-tandem mass spectrometry at Tandem LabCorp (Durham, NC, USA).

The area under the prefilter, postfilter, and systemic concentration-time curves (AUC) from 0 to $60 \mathrm{~min}$ was calculated using the linear trapezoidal rule. Overall efficiency was calculated using the $\mathrm{AUC}_{\text {last }}[($ pre - post $) /$ pre $\times 100]$. Postfilter samples with melphalan concentration values lower than the level of detection were calculated using linear interpolation. For systemic samples, linear interpolation was deemed inappropriate because some interpolated values were higher than the limit of detection $(25 \mathrm{ng} / \mathrm{mL})$. Therefore, values were set to half of the lowest limit of detection $(12.5 \mathrm{ng} / \mathrm{mL})$ with the exception of the zero time point, which was assumed to be $0 \mathrm{ng} / \mathrm{mL}$.

\section{Transfilter Pressure Gradient}

Pressure gauges were placed before and after the filter within the EC. Once the filter was online, prefilter and postfilter pressure measurements were taken immediately prior to melphalan infusion and at 3-min intervals throughout the procedure. The transfilter pressure gradient was calculated as the difference between the prefilter and postfilter pressure.

\section{Results}

All six animals successfully underwent the entire 60-min procedure. The mean EC flow rate was $530 \pm 34 \mathrm{~mL} / \mathrm{min}$.

\section{Pharmacokinetics}

In general, the prefilter melphalan concentration gradually increased over the course of the infusion period. The mean maximum concentration $\left(C_{\max }\right)$ and SD was $10,072 \pm$ $1762 \mathrm{ng} / \mathrm{mL}$, and $C_{\max }$ typically occurred between 21 and 30 min of infusion. After the infusion period, there was an immediate and continuous decrease in melphalan inlet concentration until the end of the procedure. A summary of the $\mathrm{AUC}_{\text {last }}$, overall filteration efficiency, and $C_{\max }$ is shown in Table 1 . The mean filter efficiency for the six animals during the 60 -min procedure was $99.0 \pm 0.4 \%$. The mean prefilter, postfilter, and systemic plasma melphalan concentrations are displayed in Fig. 2, and the mean plasma melphalan removal efficiency over time is shown in Fig. 3. The full data set of 
Table 1 Melphalan pharmacokinetic parameters and extraction efficiency

\begin{tabular}{|c|c|c|c|c|c|c|c|}
\hline \multirow[t]{2}{*}{ Parameters and efficiency } & \multicolumn{3}{|c|}{$\mathrm{AUC}(\min * \mathrm{ng} / \mathrm{mL})$} & \multirow{2}{*}{$\begin{array}{l}\text { Efficiency }(\%) \\
{[(\text { pre }- \text { post }) / \text { pre } \times 100]}\end{array}$} & \multicolumn{3}{|c|}{$C_{\max }(\mathrm{ng} / \mathrm{mL})$} \\
\hline & Prefilter & Postfilter & Systemic & & Prefilter & Postfilter & Systemic \\
\hline Mean & 331,933 & 3,171 & 7,921 & 99.0 & 10,072 & 91 & 265 \\
\hline $\mathrm{SD}$ & 56,039 & 873 & 5,809 & 0.4 & 1,762 & 26 & 257 \\
\hline Minimum & 262,485 & 2,266 & 3,871 & 98.5 & 8,510 & 54 & 101 \\
\hline Median & 333,548 & 3,113 & 4,721 & 99.0 & 9,525 & 102 & 128 \\
\hline Maximum & 415,215 & 4,307 & 17,912 & 99.5 & 13,400 & 119 & 751 \\
\hline
\end{tabular}

$A U C$ area under the concentration-time curve

$n=6$ animals

Fig. 2 Mean prefilter, postfilter, and systemic plasma melphalan concentrations over time. Samples were taken at procedure. Error bars represent $1 \mathrm{SD}$ from the mean 3-min intervals throughout the

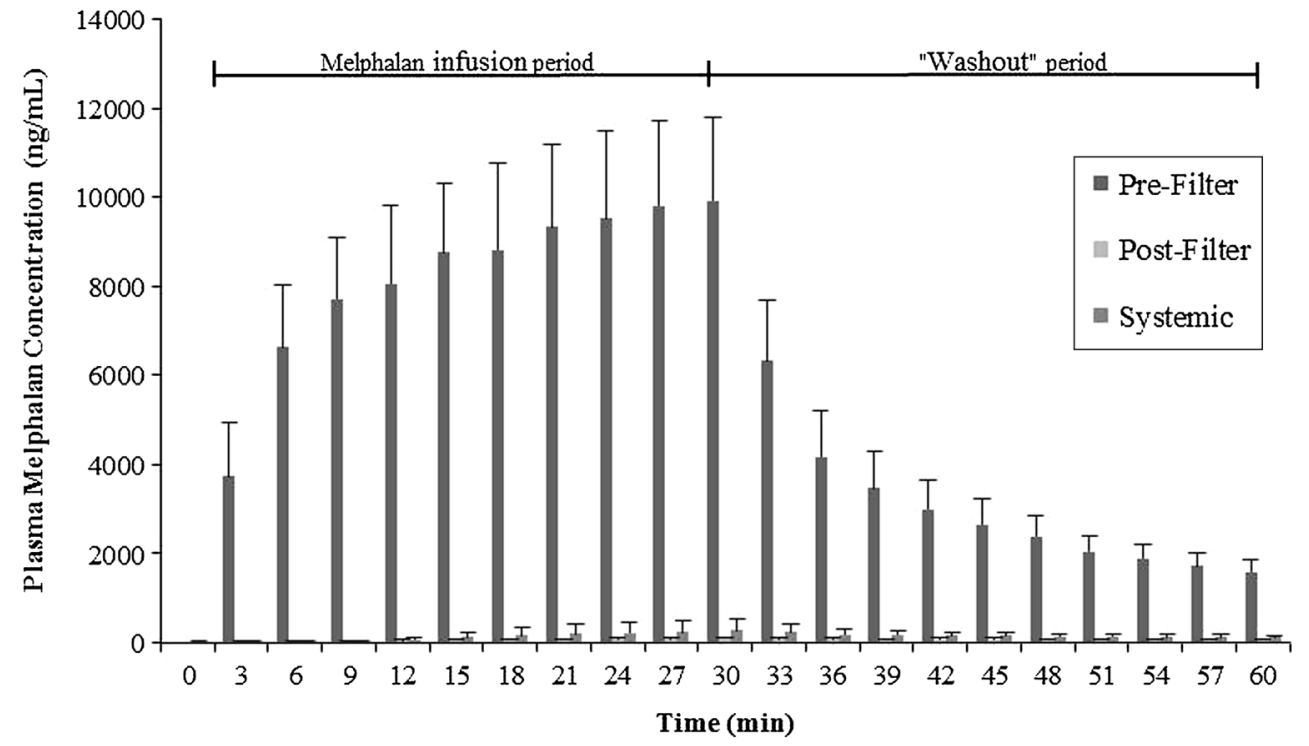

prefilter, postfilter, and systemic plasma melphalan concentration values is available in Supplemental File 1.

\section{Transfilter Pressure Gradient}

The mean pressure gradient across the filter during the entire procedure was $20.9 \pm 7.3 \mathrm{mmHg}$ with a prefilter pressure of $50.5 \pm 9.4 \mathrm{mmHg}$ and a postfilter pressure of $29.5 \pm 10.0 \mathrm{mmHg}$. The full data set of in-line pressure data are shown in Supplemental File 2.

\section{Blood Chemistry}

The following blood parameters were analyzed: hematocrit, platelet count, neutrophils, albumin, and fibrinogen. The changes in these parameters as a result of the melphalan-PHP procedure with the filter were assessed by comparing values at baseline and at $60 \mathrm{~min}$ of filtration (Table 2). The concentration of all parameters decreased between baseline and after $60 \mathrm{~min}$ of filtration. The full data set of blood chemistry data are shown in Supplemental File 3.
Fig. 3 Mean melphalan removal efficiency over time. Error bars represent $1 \mathrm{SD}$ from the mean

\section{Discussion}

PHP is a locoregional drug delivery that has been shown in clinical trials to be effective in the treatment of primary and

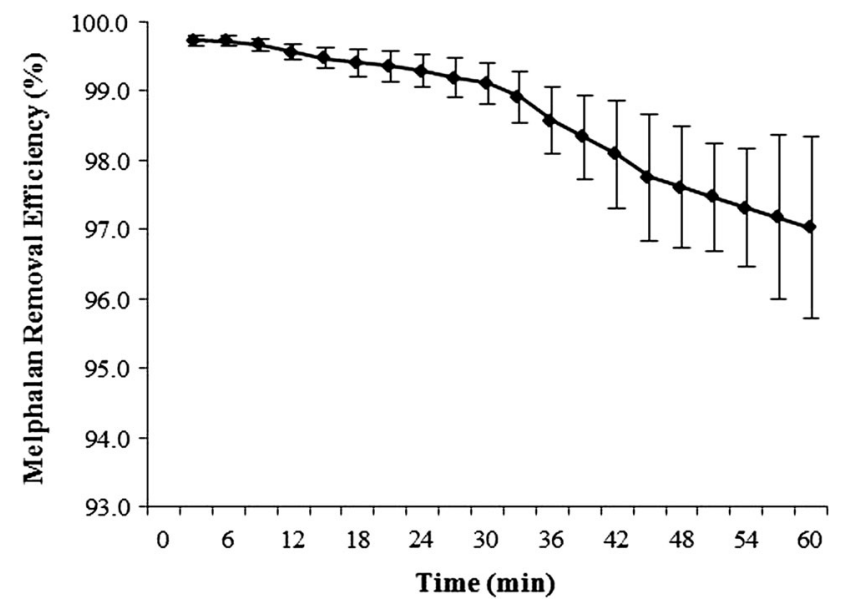


Table 2 Clinical pathology results

\begin{tabular}{llllll}
\hline Time point & Hematocrit $(\%)$ & Platelet count $\left(\times 10 . \mathrm{e}^{3} / \mu \mathrm{L}\right)$ & Neutrophils, Abs $(\times 10 . \mathrm{e} 3 / \mu \mathrm{L})$ & Albumin $(\mathrm{g} / \mathrm{dL})$ & Fibrinogen $(\mathrm{mg} / \mathrm{dL})$ \\
\hline Baseline & $30.73 \pm 1.62$ & $342.20 \pm 60.81$ & $5.07 \pm 1.21$ & $3.55 \pm 0.23$ & $226.17 \pm 32.12$ \\
$60 \mathrm{~min}$ & $23.43 \pm 3.02$ & $177.00 \pm 59.97$ & $1.83 \pm 0.25$ & $2.02 \pm 0.13$ & $142.83 \pm 12.48$ \\
\hline
\end{tabular}

All data are mean $\pm \mathrm{SD}$

$n=6$ animals

metastatic hepatic neoplasms [4, 7-9]. This technique uses a minimally invasive and repeatable approach to partially isolate the hepatic circulation and deliver high doses of chemotherapy. Notably, PHP offers two extremely important advantages compared with traditional systemic chemotherapy: (1) the ability to deliver a very high dose of cytotoxic drug specifically to a region of interest; and (2) minimization of systemic exposure resulting in fewer side effects than seen with systemic administration of high concentrations of chemotherapeutic agent.

Despite hemofiltration in PHP, during a phase I clinical trial, bone marrow suppression was still the most common dose- or treatment-limiting side effect [4]. In that study, the calculated melphalan filtration efficiency was $78.5 \pm$ $15.2 \%$ in patients receiving a dose of $3 \mathrm{mg} / \mathrm{kg}$ ideal body weight (IBW). Therefore, although the filter provided a significant protective effect, a high incidence of melphalanrelated side effects was observed. To decrease systemic side effects, a novel filter was designed and showed a melphalan extraction of $98 \%$ in this study.

The GEN 2 hemofiltration system differs from the previous filter generations in that it is comprised of two hemofiltration cartridges in a single housing containing novel, proprietary, free-floating spherical filtration media. The connections to the hemoperfusion circuit have been modified to facilitate the system setup.

The increased melphalan filtration efficiency of the filter resulted in a low systemic exposure throughout the procedure. The mean systemic AUC was 7,921 min * ng/mL. In contrast, systemic AUC in the phase I clinical trial at the $3 \mathrm{mg} / \mathrm{kg}$ IBW dose was 37,800 min * ng/mL. A comparison of the phase I study patients treated with $3 \mathrm{mg} / \mathrm{kg}$ IBW (dose mean $182 \mathrm{mg}$; range 153-212 $\mathrm{mg}$, and the animals treated in the current study $(220 \mathrm{mg})$ show that the filter inlet maximal concentrations were similar $\left(C_{\max }\right.$ of 11,820-10,072 ng/mL, respectively). The procedure and devices used were similar, with the exception of the filter; therefore the difference in the systemic AUC is attributed to filter's melphalan filtration efficiency. This increase in filter efficiency of the GEN 2 filter may provide an improved side effect profile compared with previous filters used in PHP trials.

The low systemic exposure seen in these studies raises the question as to whether greater melphalan doses could be used in the system, thus allowing for greater liver/tumor exposure while potentially maintaining or decreasing systemic exposures seen in clinical trials. The phase I study showed that although hepatotoxicity at a dose of $3 \mathrm{mg} / \mathrm{kg}$ IBW was manageable/acceptable, an increase to $3.5 \mathrm{mg} / \mathrm{kg}$ IBW resulted in a significant increase in grade $3 / 4$ hepatotoxicity [4]; thus, improved efficiency with the GEN 2 filter may not translate to increased maximum tolerated dose.

It is noteworthy that, on average, the systemic melphalan concentration was greater than the melphalan concentration at the filter outlet. The greater ratio of systemic-topostfilter concentrations with the GEN 2 filter suggest that the amount of drug not captured by the filter system does not represent the majority of drug entering the systemic circulation. One potential source of melphalan in the systemic circulation is leakage by way of perihepatic collateral vessels. Moreover, unlike the human clinical procedure, nonhepatic branches of the celiac artery were not embolized before the procedure because the goals of this acute study were to evaluate the GEN 2 filter with respect to (1) filter efficiency, (2) in-line filter pressures, and (3) extraction of blood parameters, not to perform a complete analysis of systemic exposure. Published reports of clinical studies using hepatic artery infusion and isolated hepatic perfusion procedures, which were the basis for the development of PHP, have reported average leakages of 1.6 and $18 \%$ [10, 11].

Because the study was not a survival study some end points, including bone marrow suppression and clinical histology, were not determined. It remains to be determined whether the expected decrease in systemic exposure occurs clinically and whether it will be sufficient to decrease/eliminate bone marrow suppression. Hepatotoxicity is not expected to be impacted by the GEN 2 filter because the filtration occurs after the liver has been exposed to the injected dose; however, an improved higher efficiency filter may decrease the overall exposure to the liver by decreasing the amount of drug that enters the liver from the general circulation.

Although porcine animal models have been used extensively in preclinical PHP studies, it should be noted that this model differs from the clinical situation in that it is an acute study and uses healthy juvenile animals with undiseased livers. However, it is noteworthy that the prefilter 

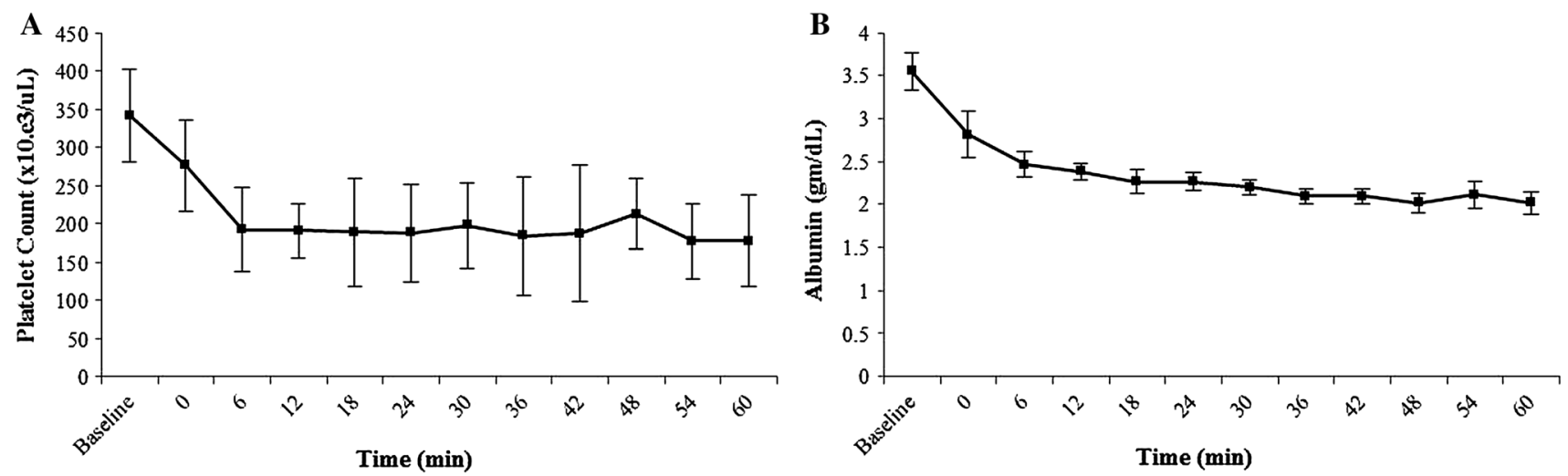

Fig. 4 Mean platelet count (A) and albumin (B) concentrations over time. Error bars represent 1 SD from the mean. C Copyright 2013 Delcath Systems, Inc. All rights reserved

melphalan concentrations observed during the study were consistent with those seen in the phase I study at $3 \mathrm{mg} / \mathrm{kg}$ IBW. The amount and concentration of melphalan presented to the filter was comparable with the clinical situation and thus relevant [4]. Measuring the transfilter pressure gradient is a means of monitoring the restriction of blood through the filter. In our experience with two previous filter generations, the transfilter pressure gradients have occasionally exceeded $200 \mathrm{mmHg}$, the maximum transfilter pressure gradient defined in the IFU for the PHP system. In all development testing and in this study, the new filter design resulted in transfilter pressure gradients $<200 \mathrm{mmHg}$, which is expected to prevent early procedure terminations resulting from low extracorporeal flow and to limit damage to blood cells.

All measured blood parameters decreased during the course of the PHP procedure, especially between baseline and the first 6 min of filtration, and nearly all adsorption occurred within 45 min after filtration started. This sharp decrease in the first 6 min is partly caused by dilution from (1) fluids administered previous and during the procedure to maintain hydrostatic pressure; (2) resident saline used to prime the filter and the EC that entered the animals' circulation when the filters were brought online; and (3) the $500 \mathrm{~mL}$ of saline that was used to deliver the drug. Together, these additional fluids contributed to the decrease in blood component concentrations during infusion, particularly between baseline and 6 min of filtration (Fig. 4). However, it is unlikely that dilution accounts for the total decrease in blood components, and it is expected that filter adsorption of components is a contributing factor. Ku et al. [12] reported similar platelet loss in a doxorubicin PHP trail, and levels returned to normal ranges in 1-2 weeks without intervention. In an isolated hepatic perfusion porcine study with tumor-necrosis factor- $\alpha$ and melphalan reported by Rinkes et al. [13], albumin level decreases were very similar to our study and also returned to normal levels within 1-2 weeks. Although the procedure has a measurable effect on the blood and serum components, it is expected that these changes would be clinically manageable using standard techniques currently used in post-PHP patient management (i.e., administration of fresh frozen plasma, albumin solution, or platelets).

In conclusion, the GEN 2 filter operates within the EC of the PHP system with very low pressure across the filter and allows for enhanced melphalan filtration efficiency in a porcine model. The clinical management required due to the extraction of blood components during the procedure is expected to be similar to that with the previous filtration systems. The improved filtration efficiency of the GEN 2 filter is expected to result in decreased melphalan systemic exposure and corresponding side effects. Future studies may build on this study by performing a full comparison of the GEN 1 and GEN 2 filtration systems and including evaluation of catecholamine extraction to provide insight into expected blood pressure management with the GEN 2 filtration system.

Acknowledgments The authors are indebted to Krishna Kandarpa for review of this manuscript. We also like to thank Brian McCormick for expertise in perfusion. This work was financially supported by Delcath Systems, Inc., New York, NY, USA.

Conflict of interest Elizabeth G. McAndrew, William M. Appling, Nicole E. Hryniewich, Kevin D. Jarvis, Steven M. Markos, Timothy P. Sheets, Rajneesh P. Uzgare, and Daniel S. Johnston, are past and present employees of Delcath Systems, Inc., New York, NY.

Open Access This article is distributed under the terms of the Creative Commons Attribution License which permits any use, distribution, and reproduction in any medium, provided the original author(s) and the source are credited. 


\section{References}

1. Alexander HR Jr, Butler CC (2010) Development of isolated hepatic perfusion via the operative and percutaneous techniques for patients with isolated and unresectable liver metastases. Cancer J 16(2):132-141

2. Ausman RK (1961) Development of a technique for isolated perfusion of the liver. N Y State J Med 61:3993-3997

3. Lin G, Lunderquist A, Hagerstrand I, Boijsen E (1984) Postmortem examination of the blood supply and vascular pattern of small liver metastases in man. Surgery 96(3):517-526

4. Pingpank JF, Libutti SK, Chang R et al (2005) Phase I study of hepatic arterial melphalan infusion and hepatic venous hemofiltration using percutaneously placed catheters in patients with unresectable hepatic malignancies. J Clin Oncol 23(15): 3465-3474

5. Van De Velde CJH, Kothuis BJL, Barenbrug HWM et al (1985) A successful technique of in vivo isolated liver perfusion in pigs. J Surg Res 41:593-599

6. Pinguet F, Culine S, Bressolle F et al (2000) A phase I and pharmacokinetic study of melphalan using a 24-hour continuous infusion in patients with advanced malignancies. Clin Cancer Res 6:57-63

7. Hwu W, Salem R, Pollak J et al (1999) A clinical-pharmacological evaluation of percutaneous isolated hepatic infusion of doxorubicin in patients with unresectable liver tumors. Oncol Res 11:529-537

8. Ku Y, Iwasaki T, Fukumoto T et al (1998) Induction of long-term remission in advanced hepatocellular carcinoma with percutaneous isolated liver chemoperfusion. Ann Surg 227(4):519-526

9. Pingpank JF, Hughes MS, Alexander HR, et al (2010) A phase III random assignment trial comparing percutaneous hepatic perfusion with melphalan (PHP-mel) to standard of care for patients with hepatic metastases from metastatic ocular or cutaneous melanoma. J Clin Oncol (Meeting Abstracts) 28(18 Suppl):LBA8512. http:// meeting.ascopubs.org/cgi/content/abstract/28/18_suppl/LBA8512

10. van Iersel LB, Gelderblom H, Vahrmeijer AL et al (2008) Isolated hepatic melphalan perfusion of colorectal liver metastases: outcome and prognostic factors in 154 patients. Ann Oncol 19(6):1127-1134

11. Marinelli A, de Brauw LM, Beerman H et al (1996) Isolated liver perfusion with mitomycin $\mathrm{C}$ in the treatment of colorectal cancer metastases confined to the liver. Jpn J Clin Oncol 26(5):341-350

12. Ku Y, Tominaga M, Iwasaki T et al (1996) Percutaneous hepatic venous isolation and extracorporeal charcoal hemoperfusion for high-dose intraarterial chemotherapy in patients with colorectal hepatic metastases. Surg Today 26(5):305-313

13. Borel Rinkes IH, de Vries MR, Jonker AM et al (1997) Isolated hepatic perfusion in the pig with TNF-alpha with and without melphalan. Br J Cancer 75(10):1447-1453 